\title{
Mental Capacity and Decision Making in Bipolar Disorder
}

\section{Gonzalo Salazar de Pablo* and Julio David Vaquerizo Serrano}

Department of Child and Adolescent Psychiatry, London, UK

*Corresponding author: Gonzalo Salazar de Pablo, Department of Child and Adolescent Psychiatry, London, UK

Keywords: Bipolar Disorder; Capacity; Decision making

\section{Introduction}

Bipolar disorder is a chronic, recurrent and potentially debilitating illness characterised by fluctuations in mood state and energy, which is associated with significant impairments in cognitive, social and everyday functioning. Difficulties appear especially in the manic or depressive phase, but this disorder allows more than half of these patients to have a normal life and make their own decisions when no severe symptoms appear, which is in these patients for most of their life.

\section{Principles}

a. All people have mental capacity until proven otherwise, even if they suffer from a mental illness.

b. An important principle in the evaluation of mental capacity is the freedom every person must make bad decisions. The fact that a person might make an irrational decision is not proof enough of incapacity. These facts may raise doubts but must not be decisive in the process

c. In order to prove inability for decision making, it is necessary to demonstrate disability, through a diagnosis of mental illness that incapacitates the abilities related to the decision-making process at that moment and for a specific task that is being evaluated.

d. When difficulties in the decision-making process appear, the least restrictive suitable and available measure should be taken. In this sense, curatorship may be more beneficial than guardianship. The best interest of the patients should be uppermost in the mind of a curator. Only in some areas and when the patient is unwell, the curator person will make decisions on economic or health aspects.

\section{Practical Issues}

a. To determine that a person does not have the capacity to make his own decisions, a mental disorder of enough severity to be unable to understand, appreciate and reason about relevant information for decision making must appear.

b. All intermediate courses are preferred rather than extreme courses. Incapacitation must be avoided as long as it is not strictly necessary and seek less drastic measures or support systems.

c. As medical and mental health professionals, it is always important to check with family members and sometimes talk to neighbors with the patient's consent, explaining your concerns and trying to win their collaboration for the patient's medical follow-up.

d. There are several ways to support patients that suffer Bipolar disorder, so that they can make the best possible decisions. They should be adapted to the mood phase in which they are at the moment. For instance, depressed patients should be encouraged to carry out their daily life activities while hypomanic patients should be encouraged to rest and be in the most quiet and calm environment available. 
(c) (i) This work is licensed under Creative

To Submit Your Article Click Here: Submit Article

DOI: $10.32474 /$ PRJFGS.2018.03.000152

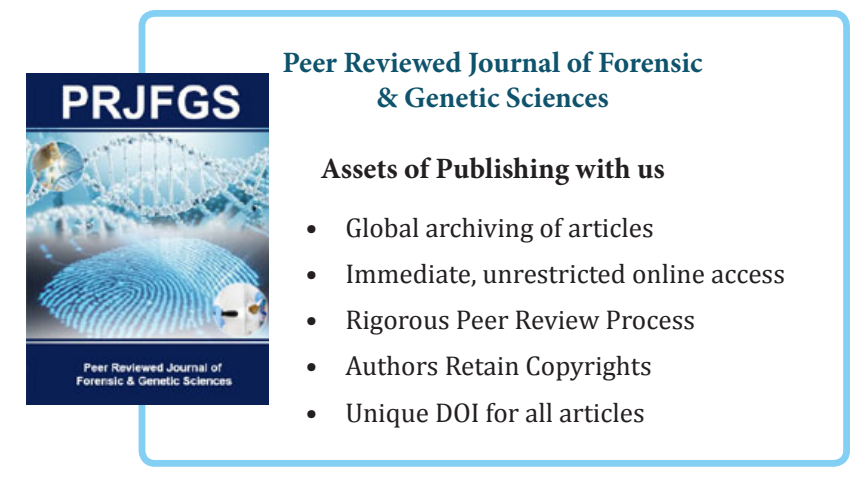

